\begin{tabular}{|c|l|}
\hline Title & Crystal structures and magnetic properties of two-dimensional antiferromagnets Co_[1-x]Zn_[x]T eMo06 \\
\hline Author(s) & Doi, Y oshihiro; Suzuki, Ryo; Hinatsu, Y ukio; Ohoyama, Kenji \\
\hline Citation & $\begin{array}{l}\text { Journal of Solid State Chemistry, 182(12), 3232-3237 } \\
\text { https://doi.org/10.1016/.jssc.2009.09.008 }\end{array}$ \\
\hline Issue Date & 2009-12 \\
\hline Doc URL & http://hdl.handle.net/2115/42487 \\
\hline Type & article (author version) \\
\hline File Information & JSSC182-12_3232-3237.pdf \\
\hline
\end{tabular}

Instructions for use 


\title{
Crystal Structures and Magnetic Properties of
}

\section{Two-Dimensional Antiferromagnets $\mathrm{Co}_{1-x} \mathrm{Zn}_{x} \mathrm{TeMoO}_{6}$}

\author{
Yoshihiro Doi, Ryo Suzuki, and Yukio Hinatsu \\ Division of Chemistry, Graduate School of Science, Hokkaido University \\ Sapporo 060-0810, Japan \\ and \\ Kenji Ohoyama \\ Institute for Materials Research, Tohoku University, \\ Sendai 980-8577, Japan
}

Yoshihiro Doi

Division of Chemistry,

Graduate School of Science,

Hokkaido University,

Sapporo 060-0810, Japan.

TEL: +81-11-706-2715

FAX: +81-11-706-4931

E-mail: doi@sci.hokudai.ac.jp 


\section{Abstract}

Crystal structures and magnetic properties of metal telluromolybdates $\mathrm{Co}_{1-x} \mathrm{Zn}_{x} \mathrm{TeMoO}_{6}(x=0.0,0.1, \ldots, 0.9)$ are reported. All the compounds have an orthorhombic structure with space group $P 2{ }_{1} 2_{1} 2$ and a charge configuration of $\mathrm{M}^{2+} \mathrm{Te}^{4+} \mathrm{Mo}^{6+} \mathrm{O}_{6}$. In this structure, $\mathrm{M}$ ions form a pseudo two-dimensional lattice in the $a b$ plane. Their magnetic susceptibility measurements have been performed in the temperature range between 1.8 and $300 \mathrm{~K}$. The end member $\mathrm{CoTeMoO}_{6}$ shows a magnetic transition at $24.4 \mathrm{~K}$. The transition temperature for solid solutions rapidly decreases with increasing $x$ and this transition disappears between $x=0.4$ and 0.5 , which is corresponding to the percolation limit for the square-planer lattice. From the magnetization, specific heat, and powder neutron diffraction measurements, it is found that the magnetic transition observed in the $\mathrm{CoTeMoO}_{6}$ is a canted antiferromagnetic ordering of $\mathrm{Co}^{2+}$ ions. The antiferromagnetic component of the ordered magnetic moment $\left(3.12(3) \mu_{\mathrm{B}}\right.$ at $\left.10 \mathrm{~K}\right)$ is along the $b$-axis. In addition, there exists a small ferromagnetic component $\left(0.28(3) \mu_{\mathrm{B}}\right)$ along the $a$-axis.

Keyword: Metal telluromolybdate; Low-dimensional magnetism; Magnetic structure; Magnetic susuceptibility; Specific heat; Neutron diffraction 


\section{Introduction}

The metal telluromolybdates $\mathrm{MTeMoO}_{6}(\mathrm{M}=\mathrm{Mg}, \mathrm{Mn}, \mathrm{Co}, \mathrm{Zn}, \mathrm{Cd})$ [1-15] have attracted many researchers due to the catalytic properties for allylic oxidation of olefins $[1,14-16]$. However, the structural information for these materials had been unknown for a long time except for the unit cell parameters and space groups. After about two decades from the first report of these compounds, Laligant determined the crystal structures for orthorhombic $\mathrm{CoTeMoO}_{6}$ and tetragonal $\mathrm{CdTeMoO}_{6}$ by the powder $\mathrm{x}$-ray diffraction, electron diffraction, and high-resolution electron microscopy measurements [11]. These compounds adopt fluorite-related structures in which the $\mathrm{M}^{2+}$ ions form a two-dimensional (2D) layer in $a b$ plane. This layer is alternatingly stacked along the c-axis with another layer consisting of $\mathrm{Mo}^{6+} \mathrm{O}_{4}$ tetrahedra and $\mathrm{Te}^{4+} \mathrm{O}_{4}$ polyhedra.

Recently we have reported the crystal structure and magnetic properties of $\mathrm{MnTeMoO}_{6}[12]$. This compound shows characteristic magnetic properties reflecting its layered structure, and the temperature dependence of magnetic susceptibility is well explained by the Heisenberg model of $S=5 / 2$ ions in a 2D square-planer lattice with an antiferromagnetic interaction $\left(\mathrm{J} / \mathrm{k}_{\mathrm{B}}=-1.96 \mathrm{~K}\right)$ between the nearest pair of Mn ions. At

$20 \mathrm{~K}$, the long-range antiferromagnetic ordering of $\mathrm{Mn}^{2+}$ magnetic moments occurs due 
to the magnetic interaction between layers; this interaction is also antiferromagnetic from the determined magnetic structure. On the other hand, the magnetic properties of the $\mathrm{CoTeMoO}_{6}$ are not well known. Only the values of effective magnetic moment (5.98 $\left.\mu_{\mathrm{B}}\right)$ and Weiss constant $(-58 \mathrm{~K})$ determined by the magnetic susceptibility $(T=100-300$ K) were reported [13], but the susceptibility data were missing in the literature. The $\mathrm{Co}^{2+}$ ion often shows a highly anisotropic magnetic behavior attributed to the residual orbital angular momentum, which is contrastive to the isotropic $\mathrm{Mn}^{2+}$ ion. Thus, it is interesting to know how this difference affects the magnetic behavior at low temperatures.

In this paper, we have studied the crystal structure and magnetic properties of $\mathrm{CoTeMoO}_{6}$ by using the powder x-ray and neutron diffraction, magnetic susceptibility, magnetization, and specific heat measurements. In order to obtain the further information concerning the two-dimensional behavior of this compound, solid solutions $\mathrm{Co}_{1-\chi} \mathrm{Zn}_{x} \mathrm{TeMoO}_{6}(x=0.1,0.2, \ldots, 0.9)$ were also investigated. 


\section{Experimental}

\section{Sample preparation}

Polycrystalline samples for $\mathrm{CoTeMoO}_{6}$ and solid solutions $\mathrm{Co}_{1-x} \mathrm{Zn}_{x} \mathrm{TeMoO}_{6}(x=$ $0.1,0.2, \ldots, 0.9)$ were synthesized by the conventional solid-state reaction. As starting materials, $\mathrm{CoO}, \mathrm{ZnO}, \mathrm{TeO}_{2}$ and $\mathrm{MoO}_{3}$ were used. Stoichiometric amounts of them were mixed in an agate mortar. The mixtures were pressed into pellets and sealed in an evacuated silica tube to prevent the loss of reagents by the volatilization, and then the ampoules were fired at $823-873 \mathrm{~K}$ for $12 \times 3 \mathrm{~h}$ with intermediate grindings and pelletings.

\section{$\underline{\text { Powder } x \text {-ray and neutron diffraction measurements }}$}

The powder x-ray diffraction (XRD) measurements were performed at room temperature in the range $10^{\circ} \leq 2 \theta \leq 120^{\circ}$ using a $2 \theta$ step size of $0.02^{\circ}$ with $\mathrm{Cu}-\mathrm{K} \alpha$ radiation on a Rigaku MultiFlex diffractometer. Powder neutron diffraction (ND) profiles were also measured for $\mathrm{CoTeMoO}_{6}$ at $10,80 \mathrm{~K}$ and room temperature in the range $3^{\circ} \leq 2 \theta \leq 153^{\circ}$ at intervals of $0.02^{\circ}$ with the wavelength of $1.81424 \AA$. Measurements were performed by the Kinken powder diffractometer for high efficiency and high resolution measurements, HERMES, of the Institute for Materials Research 
(IMR), Tohoku University [17], installed at the JRR-3M reactor in Japan Atomic Energy Agency (JAEA), Tokai. The XRD and ND data were analyzed by the Rietveld technique, using the programs RIETAN2000 [18] and FullProf [19], respectively.

\section{Magnetic susceptibility and specific heat measurements}

The temperature dependence of the magnetic susceptibilities was measured under both zero-field-cooled (ZFC) and field-cooled (FC) conditions in an applied field of 0.1 T over the temperature-range 1.8-400 K using SQUID magnetometer (Quantum Design, MPMS-5S). For the $\mathrm{CoTeMoO}_{6}$, the field dependence of magnetization at $5 \mathrm{~K}$ in the field-range of $-5 \mathrm{~T} \leq H \leq 5 \mathrm{~T}$ and the remanent magnetization measurements were also performed. For the latter measurement, the sample was cooled down to $5 \mathrm{~K}$, and the magnetic field was applied up to $5 \mathrm{~T}$ and then reduced to zero. After that, the data were collected in the temperature range from 5 to $40 \mathrm{~K}$.

In addition, the specific heat measurement for $\mathrm{CoTeMoO}_{6}$ was carried out using a relaxation technique with a commercial physical property measurement system (Quantum Design, PPMS model) in the temperature range $1.8-300 \mathrm{~K}$. The sintered sample in the form of a pellet was mounted on a thin alumina plate with grease for better thermal contact. 


\section{Results and discussion}

\section{Crystal structure}

Polycrystalline samples of $\mathrm{Co}_{1-x} \mathrm{Zn}_{x} \mathrm{TeMoO}_{6}(x=0.0,0.1, \ldots, 0.9)$ were obtained as single-phase materials. The x-ray diffraction profiles were indexed with an orthorhombic unit cell $\left(a \sim 5.3 \AA, b \sim 5.1 \AA\right.$, and $c \sim 8.9 \AA$ ), space group $P 2{ }_{1} 2{ }_{1} 2$ (No. 18) for all the compounds. The structural refinements were performed by the Rietveld method using a structural model for $\mathrm{CoTeMoO}_{6}$ [11], and all the data showed a good agreement between observed and calculated intensities. The diffraction patterns for $x=$ 0.0 and 0.5 , and the schematic crystal structure are shown in Figs. 1 and 2, respectively. The variation of lattice parameters with $x$ of $\mathrm{Co}_{1-x} \mathrm{Zn}_{x} \mathrm{TeMoO}_{6}$ is plotted in Fig. 3. The monotonous variation (increasing for $a$ and $c$; decreasing for $b$ ) with $x$ indicates that this system form a solid solution over the range of $0 \leq x \leq 1$.

The powder neutron diffraction measurements were carried out for $\mathrm{CoTeMoO}_{6}$, and the diffraction profiles are shown in Fig. 4. The nuclear Bragg peaks observed in the data at $10,80 \mathrm{~K}$, and room temperature suggest that this compound keeps the orthorhombic structure down to $10 \mathrm{~K}$, i.e., no evidence for a structural phase transition is found. The profile at $10 \mathrm{~K}$ shows the magnetic Bragg peaks indicating the occurrence of the long-range magnetic ordering, which will be discussed later. The refined 
structural parameters are summarized in Table 1, and bond lengths and bond valence sums (BVSs) calculated from these parameters are listed in Table 2. The values of BVS indicate that the valence state of this compound is $\mathrm{Co}^{2+} \mathrm{Te}^{4+} \mathrm{Mo}^{6+} \mathrm{O}_{6}$. In this crystal structure, the $\mathrm{Co}^{2+}$ ion is located in the $(6+2)$-coordinated site with six shorter $\mathrm{Co}-\mathrm{O}$ lengths $(2.1-2.3 \AA)$ and two longer Co-O lengths $(\sim 2.9 \AA)$. Its coordination polyhedron can be represented as a distorted $\mathrm{CoO}_{6}$ octahedron (Fig. 2), which connects with each other by sharing the corner oxygen (O3) ion. In this linkage, the Co ions form a pseudo square-planner lattice in $a b$-plane (the Co-Co distance: $3.66 \AA$ ) even though there is a small shift of $\pm 0.2 \AA$ from the horizontal level. The Mo and Te ions are coordinated by four oxygen ions; the former makes a normal tetrahedron, but the latter makes a characteristic polyhedron reflecting the existence of lone pair electrons of $\mathrm{Te}^{4+}$. Similar coordination was found in $\mathrm{Co}_{6} \mathrm{Te}_{5} \mathrm{O}_{16}$ [20] and $\mathrm{Ln}_{2} \mathrm{Te}_{4} \mathrm{O}_{11}(\mathrm{Ln}=$ lanthanides) [21].

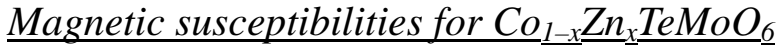

The temperature dependence of the inverse ZFC magnetic susceptibility for $\mathrm{Co}_{1-x} \mathrm{Zn}_{x} \mathrm{TeMoO}_{6}(x=0.0,0.5,0.8)$ is plotted in Fig. 5 . The data above $100 \mathrm{~K}$ show a good linearity; thus, they were fitted by the modified Curie-Weiss law: $\chi_{\mathrm{M}}=C /(T-\theta)$ $+\chi_{\text {TIP. }}$ The $\chi_{\text {TIP }}$ means the temperature-independent paramagnetic susceptibility 
$\left(5-7 \times 10^{-4} \mathrm{emu} \mathrm{mol}^{-1}\right)$. The obtained effective magnetic moments $\left(\mu_{\mathrm{eff}}\right)$ calculated from the Curie constant $(C)$ and Weiss constants $(\theta)$ are plotted in Fig. 6. The values of $\mu_{\mathrm{eff}}$ per Co ion are almost constant $\left(4.35-4.48 \mu_{\mathrm{B}}\right)$ throughout the composition range, and these values are reasonable for the $\mathrm{Co}^{2+}$ ion in the high-spin state with contribution from the orbital angular momentum [22]. The Weiss constants of $\mathrm{CoTeMoO}_{6}$ is determined to be $-58.1(9) \mathrm{K}$, which suggests hat the predominant magnetic interaction between Co ions is antiferromagnetic. The $|\theta|$ values monotonically decrease with increasing $x$ due to the magnetic dilution by doping the diamagnetic $\mathrm{Zn}^{2+}$ ion.

Figure 7 represents the magnetic susceptibilities for $\mathrm{Co}_{1-x} \mathrm{Zn}_{x} \mathrm{TeMoO}_{6}(x=0.0,0.1$, $\ldots, 0.5)$ at low temperature region. It is found that the compounds with $x \leq 0.3$ show a magnetic anomaly with the divergence between the ZFC and FC susceptibilities. For $x=$ 0.4 compound, only an onset of the divergence is observed at $2.0 \mathrm{~K}$, and above this $x$ value no magnetic anomaly has been found. As will be discussed later, this anomaly is due to an antiferromagnetic ordering of the $\mathrm{Co}^{2+}$ magnetic moments with a small spin canting. Thus, the onset of the divergence, i.e., the temperature at which a small ferromagnetic component derived from the canting antiferromagnetic ordering appears, is the Néel point in these compounds. The variation of the Néel temperature $\left(T_{N}\right)$ is plotted in Fig. 6(c). The $T_{\mathrm{N}}$ decreases quickly with increasing $x$ and the magnetic 
transition disappears between $x=0.4$ and 0.5 even though the concentration of the magnetic ion is still $>50 \%$. Such a behavior is characteristic for the low-dimensional magnetic system, and the critical point for $\mathrm{Co}_{1-x} \mathrm{Zn}_{x} \mathrm{TeMoO}_{6}$ shows a good agreement with a percolation limit calculated for the square-planer lattice (the critical concentration of magnetic ions is 0.59) [23].

\section{$\underline{\text { Magnetic properties of } \mathrm{CoTeMoO}} 6$}

The field dependence of the magnetization measured at $5 \mathrm{~K}$ and the temperature dependence of the remanent magnetization for $\mathrm{CoTeMoO}_{6}$ are plotted in Figs. 8 and 9, respectively. It is found that this compound shows a small hysteresis loop indicating the existence of a ferromagnetic component. Its saturation magnetization is $0.23 \mu_{\mathrm{B}}$. The remanent magnetization decreases with increasing temperature and reaches zero at around $T_{\mathrm{N}}$. These facts suggest that the observed ferromagnetic component is derived from the antiferromagnetic ordering of the $\mathrm{Co}^{2+}$ ion, i.e., there exists a small canting of ordered magnetic moments. The canting antiferromagnetism is often observed in compounds with lower crystal symmetry, which is caused by the Dzyaloshinsky-Moriya (D-M) interaction. The isostructural compound $\mathrm{MnTeMoO}_{6}$ also shows an antiferromagnetic transition with a slight ferromagnetic moment $\left(\sim 10^{-4} \mu_{\mathrm{B}}\right)[12]$. The 
difference in the magnitude of ferromagnetic component between $\mathrm{CoTeMoO}_{6}$ and $\mathrm{MnTeMoO}_{6}$ may reflect the difference in the $g$-factor anisotropy between $\mathrm{Co}^{2+}$ and $\mathrm{Mn}^{2+}$ ions because the strength of the D-M interaction is proportional to $(g-2) / g$ [22]. The $\mathrm{Co}^{2+}\left(3 \mathrm{~d}^{7}\right)$ ion in the octahedral crystal field often shows large $g$ values $[22,24]$ while the value of the $\mathrm{Mn}^{2+}\left(3 \mathrm{~d}^{5}\right)$ ion is normally $\sim 2$; in the latter this interaction is expected to become negligibly weak.

Figure 10(a) shows the temperature dependence of the specific heat divided by temperature $\left(C_{\mathrm{p}} T^{-1}\right)$ for $\mathrm{CoTeMoO}_{6}$. An anomaly indicating the long-range magnetic ordering of $\mathrm{Co}^{2+}$ ions is found at $24.4 \mathrm{~K}$, which corresponds to the result of the magnetic susceptibility and remanent magnetization measurements. The magnetic contribution of the data was estimated by subtracting the lattice contribution (the data for isostructural and diamagnetic $\left.\mathrm{ZnTeMoO}_{6}[12]\right)$ from the experimental data of the $\mathrm{CoTeMoO}_{6}$, and the magnetic entropy $\left(S_{\mathrm{mag}}\right)$ was calculated by $S_{\mathrm{mag}}=\int\left(C_{\mathrm{mag}} \cdot T^{-1}\right) d T$; they are plotted in Fig. 10(b). Above $T_{\mathrm{N}}$, the peak of $C_{\mathrm{mag}} T^{-1}$ shows a long tail up to $\sim 80 \mathrm{~K}$, and the magnetic entropy at $T_{\mathrm{N}}$ is $2.8 \mathrm{~J} \mathrm{~mol}^{-1} \mathrm{~K}^{-1}$ much smaller than $R \ln 4=11.53 \mathrm{~J} \mathrm{~mol}^{-1} \mathrm{~K}^{-1}$ expected from the fourfold-degenerated ground state of the high-spin $3 \mathrm{~d}^{7}$ ion. These results indicate that most of the magnetic entropy is lost before reaching $T_{\mathrm{N}}$, which may be due to the short-range magnetic ordering in the $a b$ plane. 
Powder neutron diffraction profile for $\mathrm{CoTeMoO}_{6}$ shows additional low-angle peaks below the magnetic transition temperature (Fig. 4(b)). These peaks indicate that the long-range antiferromagnetic ordering of the $\mathrm{Co}^{2+}$ magnetic moments occurs, and can be indexed using a propagation vector $\boldsymbol{k}=(0,0,1 / 2)$. This vector is the same as that found in the $\mathrm{Mn}$ analogue, $\mathrm{MnTeMoO}_{6}$ [12]; thus the antiferromagnetic structure of the Mn compound was tested for $\mathrm{CoTeMoO}_{6}$ as a starting model and finally it was found that a non-collinear antiferromagnetic structure with a small ferromagnetic component brings about a good calculation result. The determined magnetic structure of $\mathrm{CoTeMoO}_{6}$ is illustrated in Fig. 11, and the ordered magnetic moments of Co ions are shown in Table 3. The magnetic unit cell is represented as $a_{\mathrm{mag}}=a, b_{\mathrm{mag}}=b$, and $c_{\mathrm{mag}}=2 c$. The total ordered moment for $\mathrm{Co}^{2+}$ ion is determined to be $3.13(3) \mu_{\mathrm{B}}$, which is reasonable for the high spin state of the $3 \mathrm{~d}^{7}$ ion $(S=3 / 2)$. Its major component orders antiferromagnetically and the direction is along the $b$-axis, which is corresponding to the magnetic structure of $\mathrm{MnTeMoO}_{6}$ [12]. In addition, a small ferromagnetic component along the $a$-axis exists in $\mathrm{CoTeMoO}_{6}$, and this moment is comparable to that observed in the magnetic measurements. 


\section{Summary}

Crystal structures and magnetic properties of metal telluromolybdates $\mathrm{Co}_{1-x} \mathrm{Zn}_{x} \mathrm{TeMoO}_{6}(x=0.0,0.1, \ldots, 0.9)$ were investigated. These compounds adopt an orthorhombic structure with space group $P 2{ }_{1} 2_{1} 2$, and form a solid solution over the whole composition range. The magnetic susceptibility and specific heat for an end member $\mathrm{CoTeMoO}_{6}$ show an anomaly at $24.4 \mathrm{~K}$ due to an antiferromagnetic ordering of

$\mathrm{Co}^{2+}$ ions. The result of magnetically dilution by doping the diamagnetic $\mathrm{Zn}^{2+}$ ion indicates that these compounds have a characteristic as two-dimensional magnets reflecting the structural feature. The weak ferromagnetic behavior associated with the antiferromagnetic ordering was found in Co-rich compounds. From the powder neutron diffraction measurement for $\mathrm{CoTeMoO}_{6}$, this ferromagnetic moment is derived from the small canting of the antiferromagnetic ordering.

\section{Acknowledgements}

This research was partially supported by the Global COE Program (Project No. B01: Catalysis as the Basis for Innovation in Materials Science) from Ministry of Education, Culture, Sports, Science and Technology. 


\section{References}

[1] P. Forzatti and P. Tittarelli, J. Solid State Chem., 33, 421-427 (1980).

[2] I.L. Botto and E.J. Baran, Z. Anorg. Allg. Chem., 468, 221-227 (1980).

[3] P. Forzatti and F. Trifirò, Gazz. Chim. Ital., 107, 259-261 (1977).

[4] R. Kozłowski and J. Słoczyński, J. Solid State Chem., 18, 51-55 (1976).

[5] P. Forzatti, Gazz. Chim. Ital., 108, 73-75 (1978).

[6] J. Słoczyński and B. Śliwa, Z. Anorg. Allg. Chem., 438, 295-304 (1978).

[7] P. Forzatti and F. Trifirò, Gazz. Chim. Ital., 107, 35-37 (1977).

[8] P. Forzatti and G. Tieghi, J. Solid State Chem., 25, 387-390 (1978).

[9] G. Tieghi and P. Forzatti, J. Appl. Cryst., 11, 291-292 (1978).

[10] H. Hayashi, N. Kokawa, T. Moriga, S. Sugiyama, and K. Koto, J. Mol. Catal. A: Chem., 145, 301-307 (1999).

[11] Y. Laligant, J. Solid State Chem., 160, 401-408 (2001).

[12] Y. Doi, R. Suzuki, Y. Hinatsu, and K. Ohoyama, J. Phys.: Condens. Matter, 21, 046006 (2009).

[13] I.L. Botto, E.J. Baran, and G. Minelli, Solid State Commun., 50, 693-695 (1984).

[14] P. Forzatti, F. Trifirò, and P.L. Villa, J. Catal., 55, 52-57 (1978).

[15] P. Forzatti and F. Trifirò, React. Kinet. Catal. Lett., 10, 275-280 (1979).

[16] H. Hayashi, S. Sugiyama, N. Kokawa, and K. Koto, Appl. Surface Sci., 121/122, 378-381 (1997).

[17] K. Ohoyama, T. Kanouchi, K. Nemoto, M. Ohashi, T. Kajitani, and Y. Yamaguchi, Japan. J. Appl. Phys., 37, 3319-3326 (1998).

[18] F. Izumi and T. Ikeda, Mater. Sci. Forum, 321-324, 198-203 (2000). 
[19] J. Rodrigues-Carvajal, Physica B, 192, 55-69 (1993).

[20] M. Trömel and Th. Scheller, Z. Anorg. Allg. Chem., 427, 229-234 (1976).

[21] H. Mayer and M. Weil, Z. Anorg. Allg. Chem., 629, 1068-1072 (2003).

[22] R.L. Carlin, "Magnetochemistry” (Springer Verlag, Berlin) (1986).

[23] L.J. de Jongh (ed), "Magnetic Properties of Layered Transition Metal Compounds" (Kluwer Academic Publishers, Dordrecht) (1989).

[24] A. Abragam and M.H.L. Pryce, Proc. Roy. Soc. A, 206, 173-191 (1951). 


\section{Figure cations}

Fig. 1 Powder x-ray diffraction profiles for $\mathrm{Co}_{1-x} \mathrm{Zn}_{x} \mathrm{TeMoO}_{6}$ (a) $x=0.0$ and (b) $x=$ 0.5. The calculated and observed diffraction profiles are shown on the top as a solid line and cross markers, respectively. The vertical markers show positions calculated from Bragg reflections. The bottom trace is a plot of the difference between the calculated and observed intensities.

Fig. 2 Schematic crystal structure of $\mathrm{Co}_{1-\chi} \mathrm{Zn}_{x} \mathrm{TeMoO}_{6}$.

Fig. 3 Variation of lattice parameters of $\mathrm{Co}_{1-x} \mathrm{Zn}_{x} \mathrm{TeMoO}_{6}$ with $x$ value.

Fig. 4 Powder neutron diffraction profiles for $\mathrm{CoTeMoO}_{6}$ (a) at room temperature and (b) at $10 \mathrm{~K}$. The calculated and observed diffraction profiles are shown on the top as a solid line and cross markers, respectively. The upper vertical markers show positions calculated from nuclear Bragg reflections, and in (b) the lower ones show those from magnetic Bragg reflections. The bottom trace is a plot of the difference between the calculated and observed intensities.

Fig. 5 Temperature dependence of the inverse ZFC magnetic susceptibilities for $\mathrm{Co}_{1-x} \mathrm{Zn}_{x} \mathrm{TeMoO}_{6}(x=0.0,0.5$, and 0.8$)$. The solid lines represent the fitting curves by the Curie-Weiss law.

Fig. 6 Variation of (a) the effective magnetic moment $\mu_{\text {eff, }}$ (b) Weiss constant $\theta$, and (c) Néel temperature $T_{\mathrm{N}}$ for $\mathrm{Co}_{1-x} \mathrm{Zn}_{x} \mathrm{TeMoO}_{6}$ with $x$ value. An arrow in (c) 
means the percolation limit calculated for the square-planer lattice [23].

Fig. 7 Temperature dependence of the ZFC and FC magnetic susceptibilities for $\mathrm{Co}_{1-x} \mathrm{Zn}_{x} \mathrm{TeMoO}_{6}(x=0.0-0.5)$.

Fig. 8 Field dependence of the magnetization at $5 \mathrm{~K}$ for $\mathrm{CoTeMoO}_{6}$.

Fig. 9 Temperature dependence of remanent magnetization for $\mathrm{CoTeMoO}_{6}$.

Fig. 10 Temperature dependence of (a) the specific heat divided by temperature and (b) the magnetic specific heat divided by temperature and magnetic entropy for $\mathrm{MnTeMoO}_{6}$

Fig. 11 Magnetic structure of $\mathrm{CoTeMoO}_{6}$. The arrows represent the direction of magnetic moments for $\mathrm{Co}^{2+}$ ions. Diamagnetic ions are omitted. 


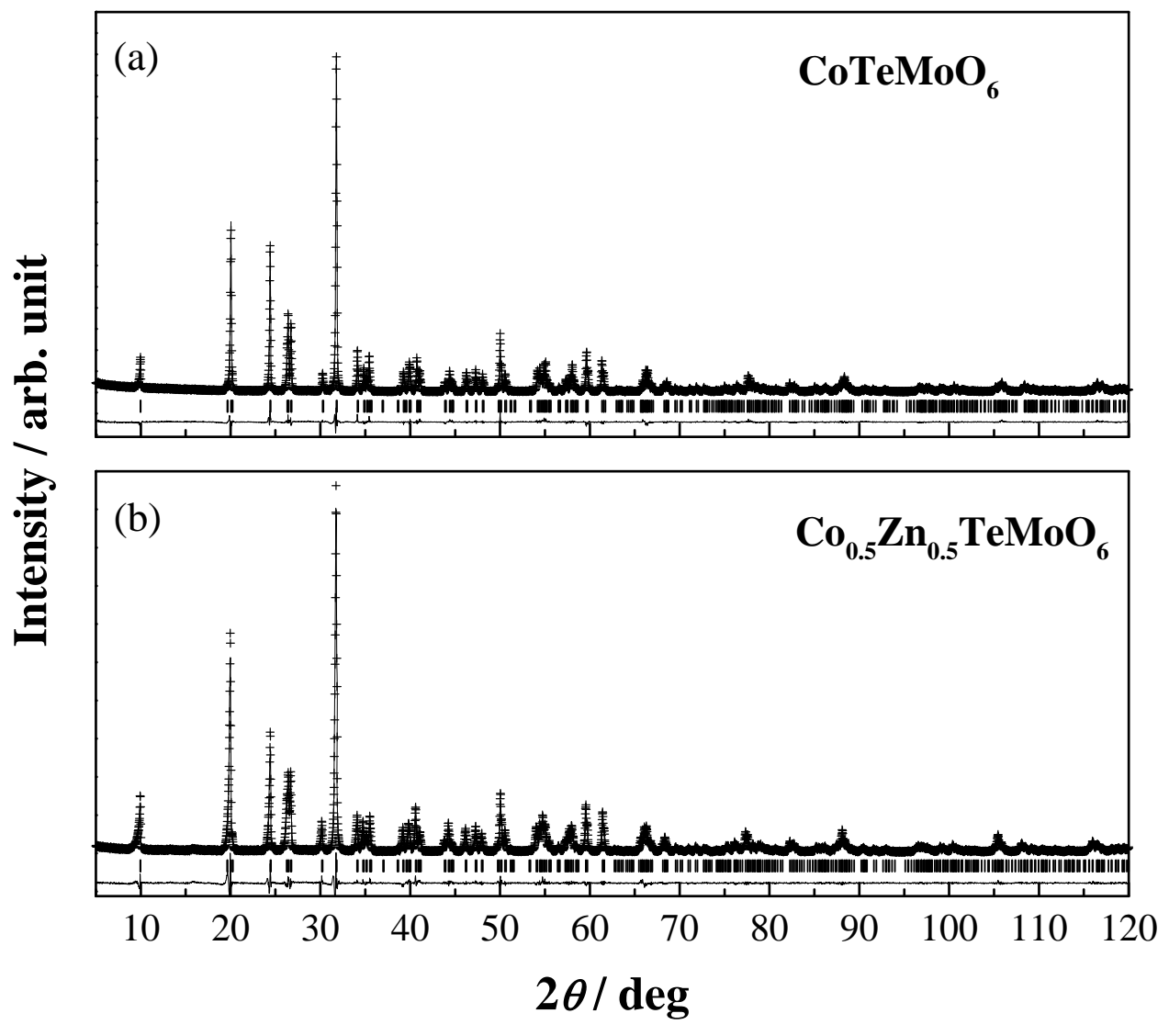

Figure 1 Doi et. al 


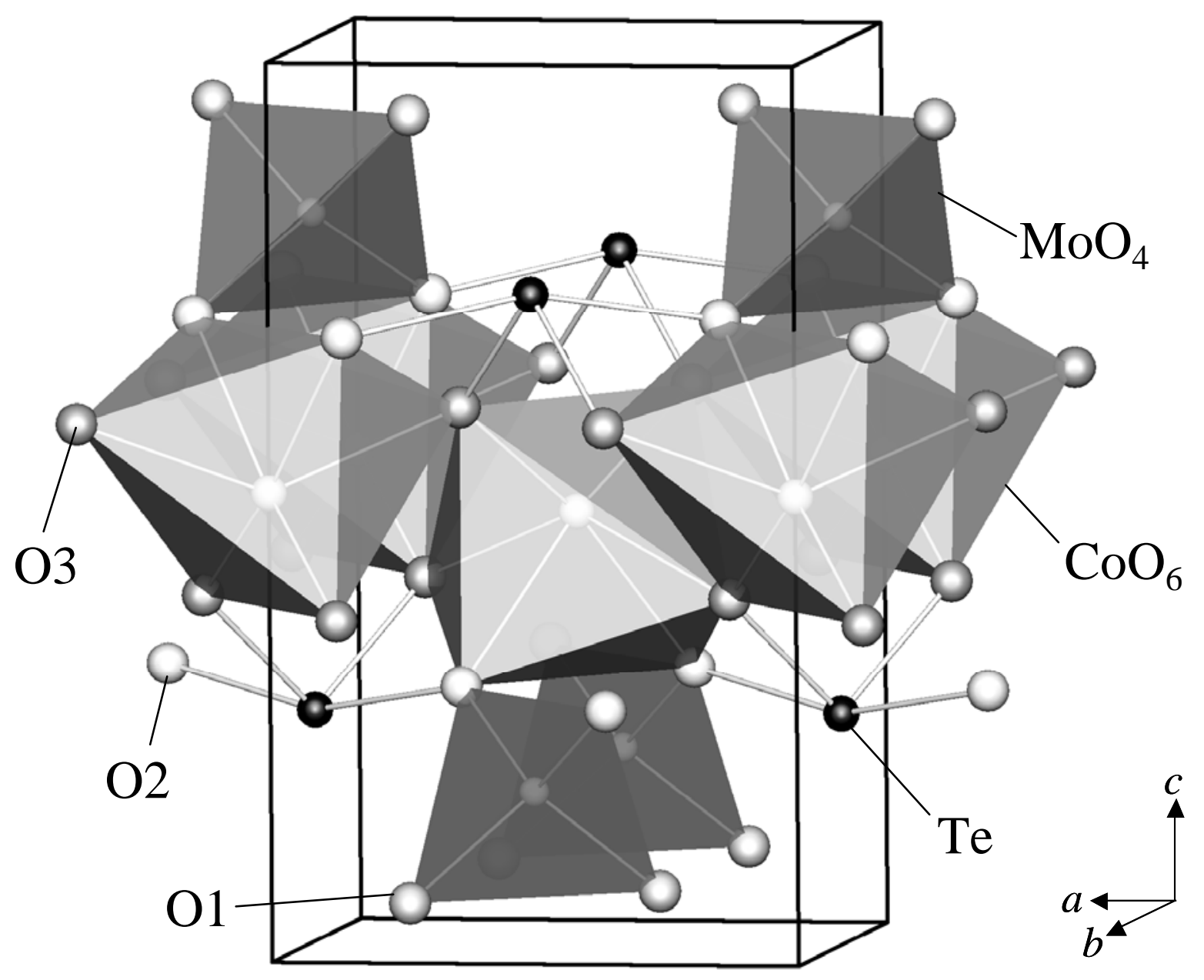

Figure 2 Doi et. al 


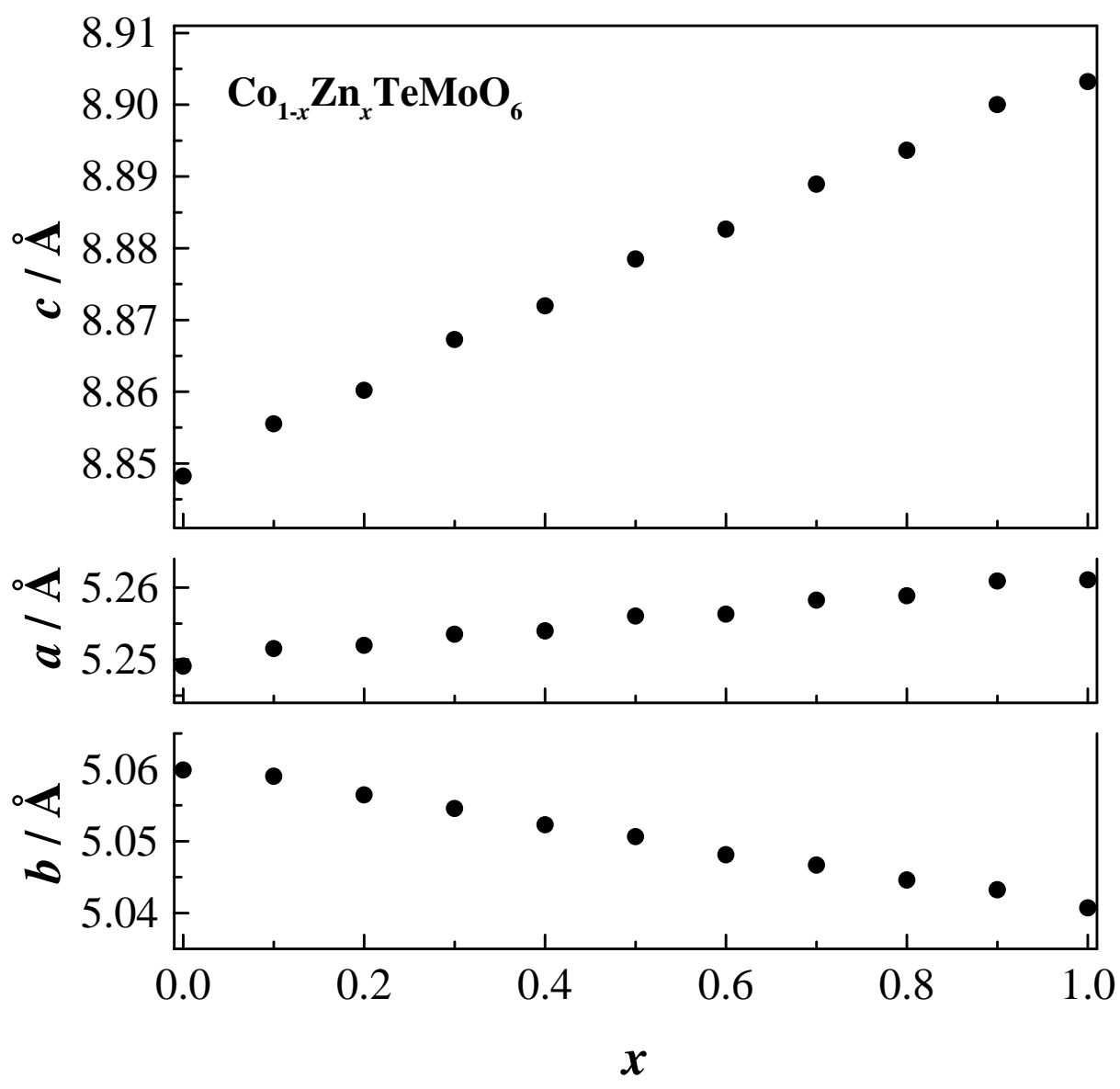

Figure 3 Doi et. al 


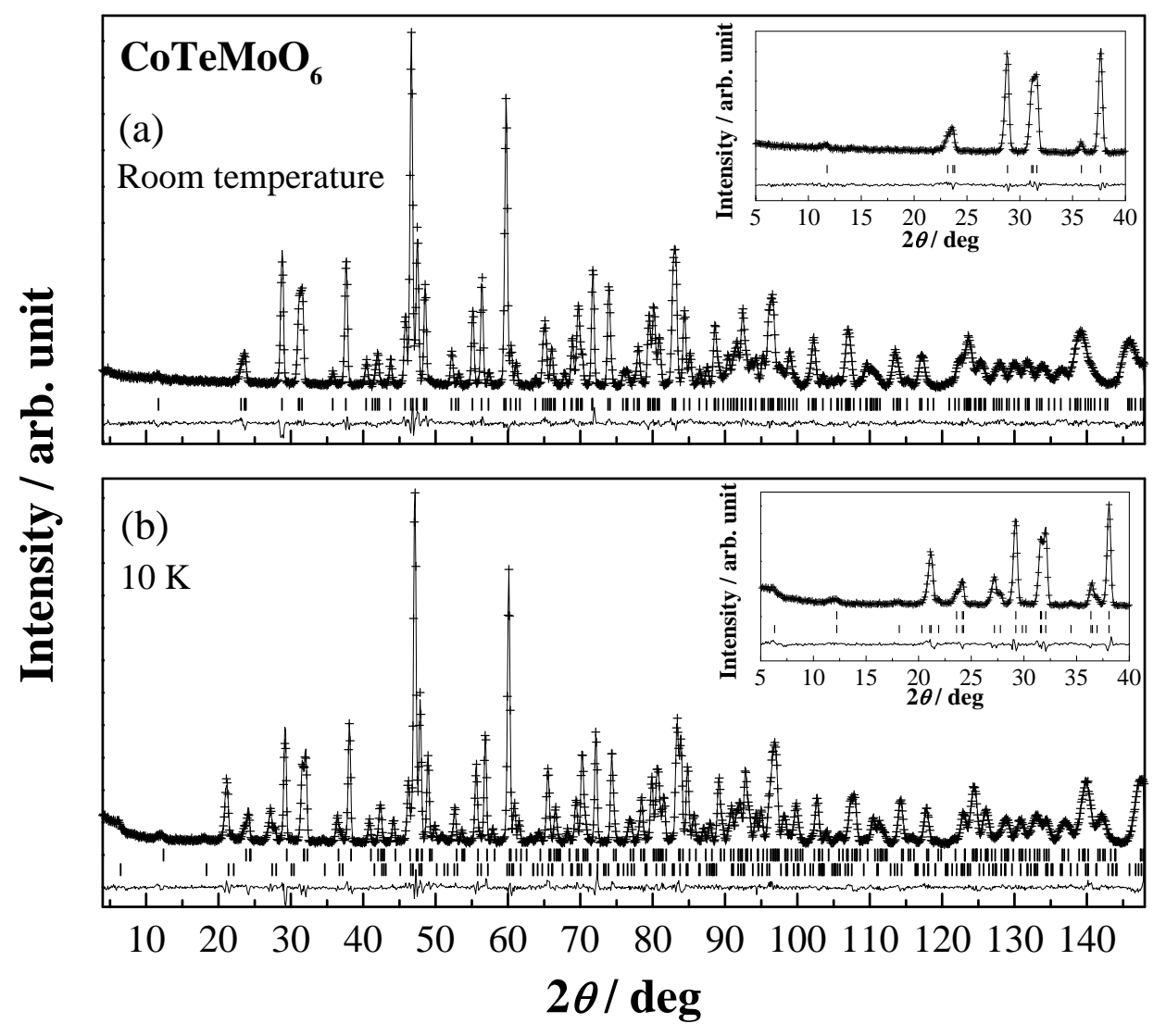

Figure 4 Doi et. al 


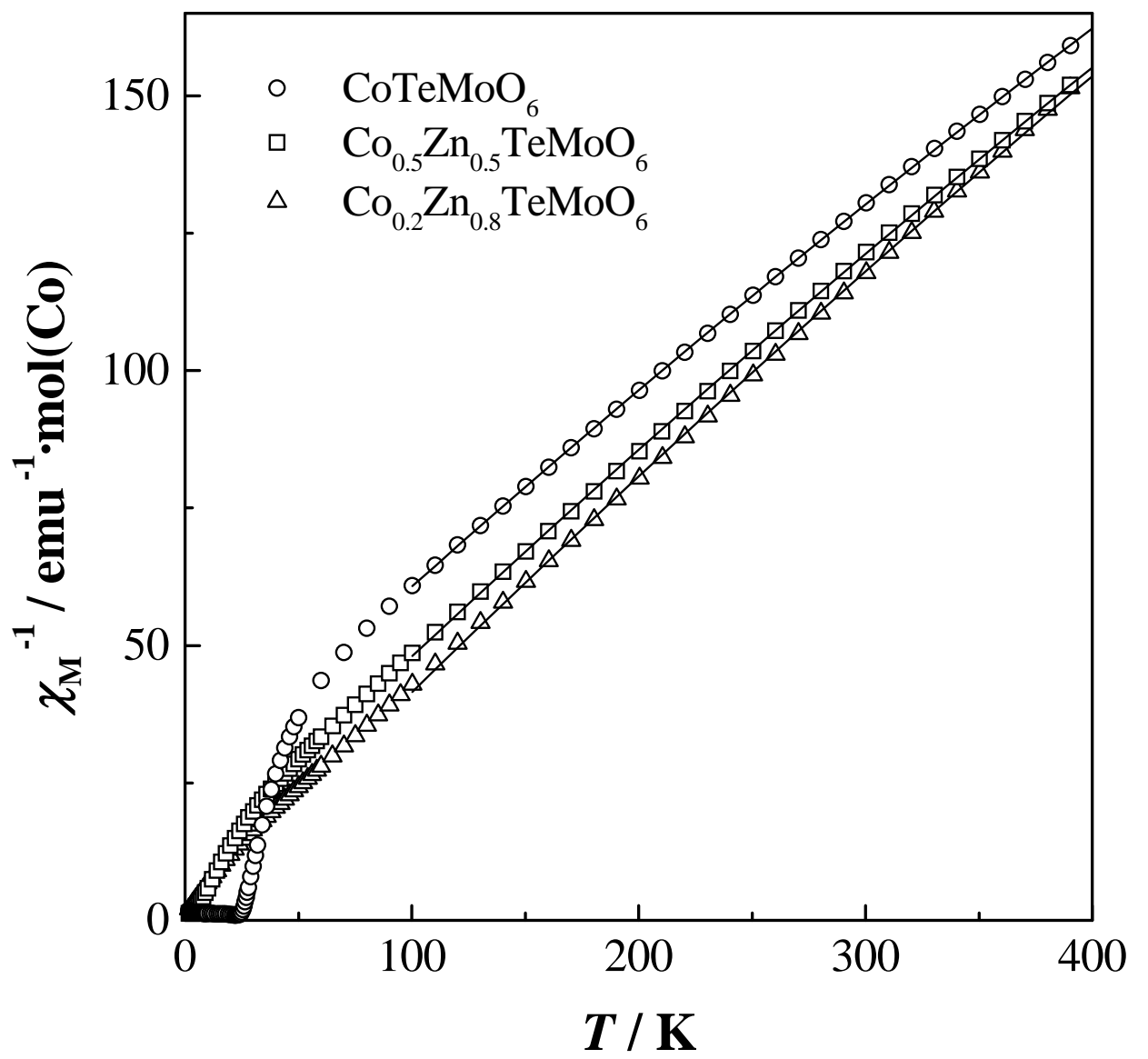

Figure 5 Doi et. al 

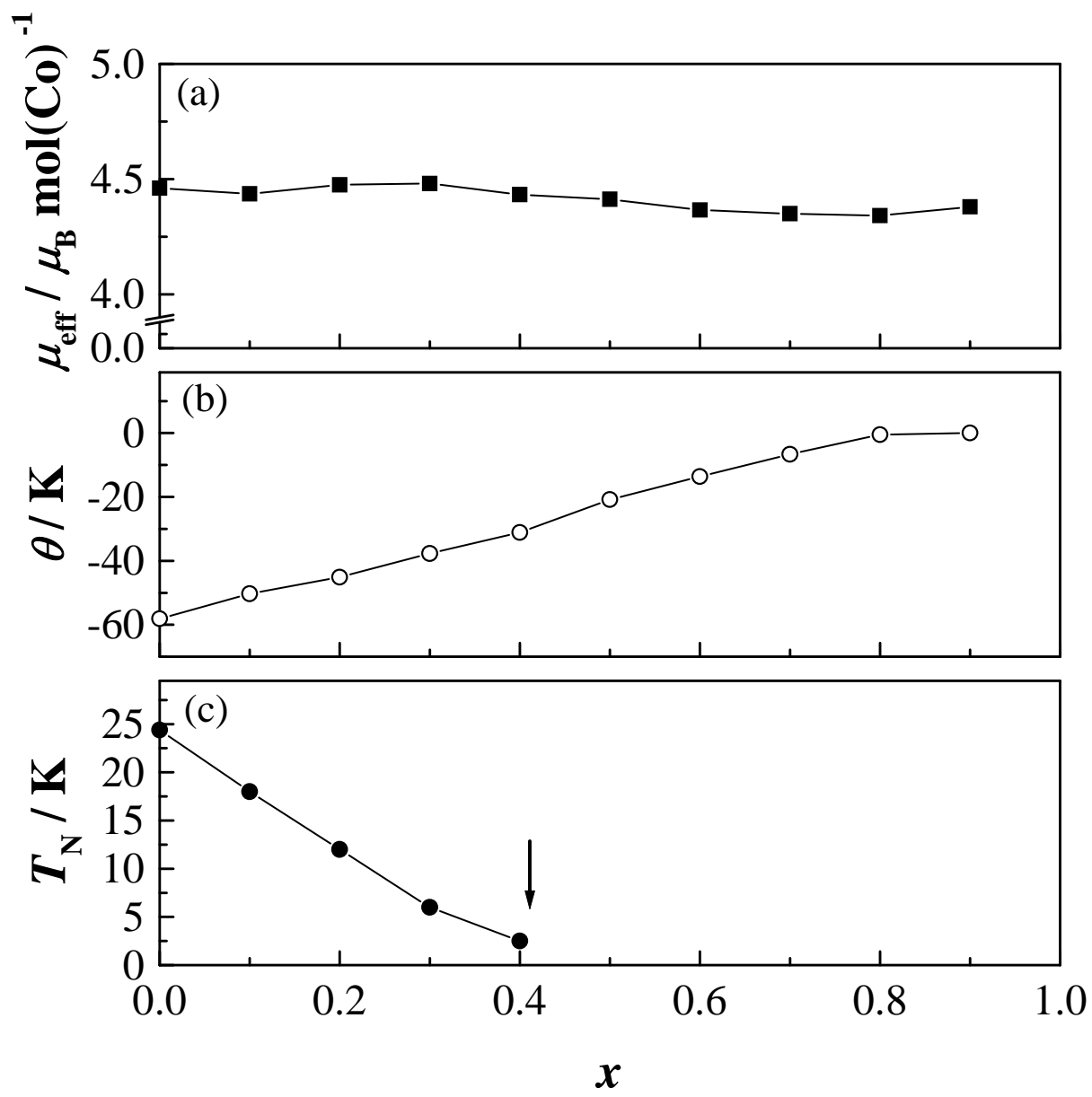

Figure 6 Doi et. al 


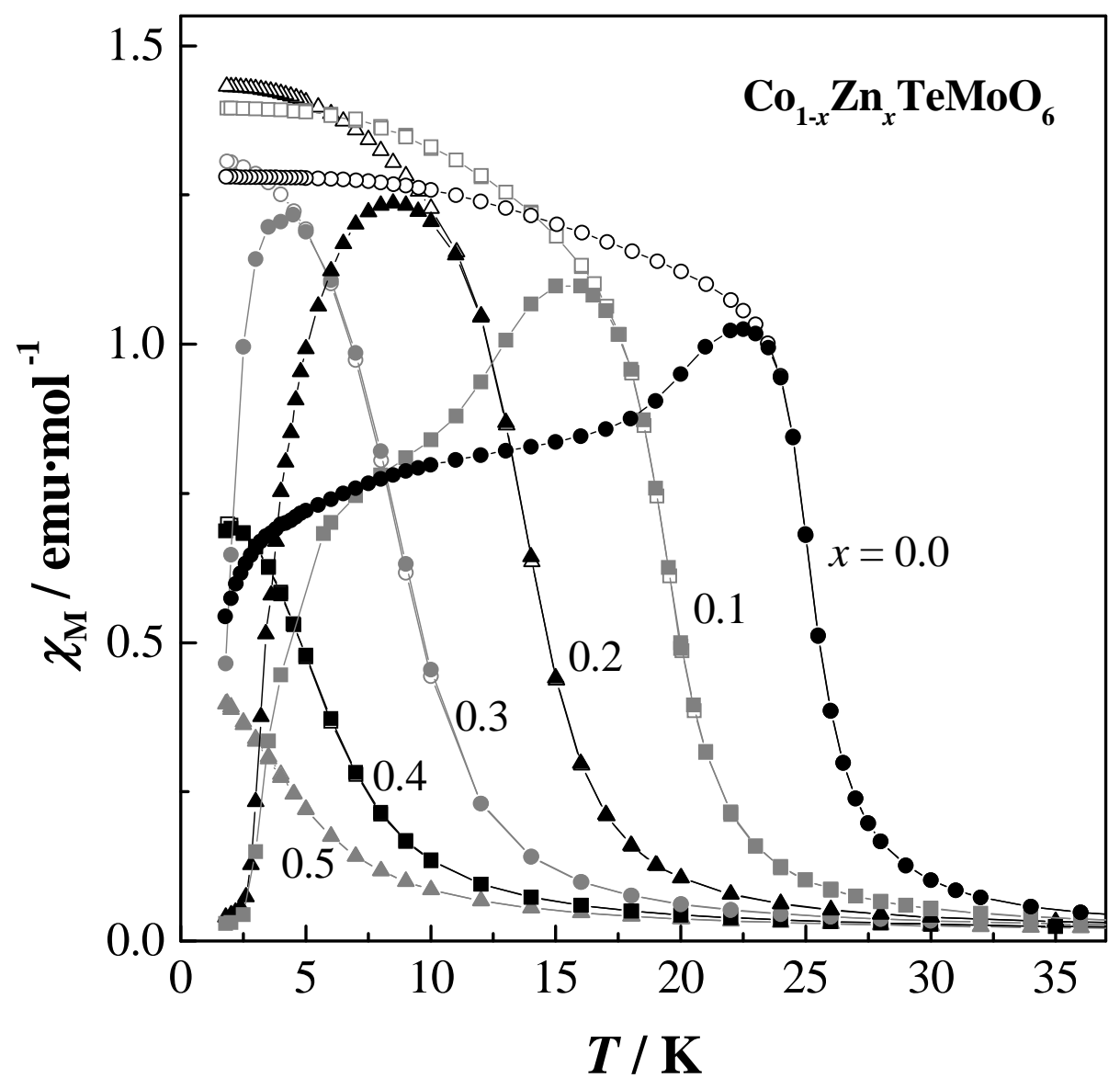

Figure 7 Doi et. al 


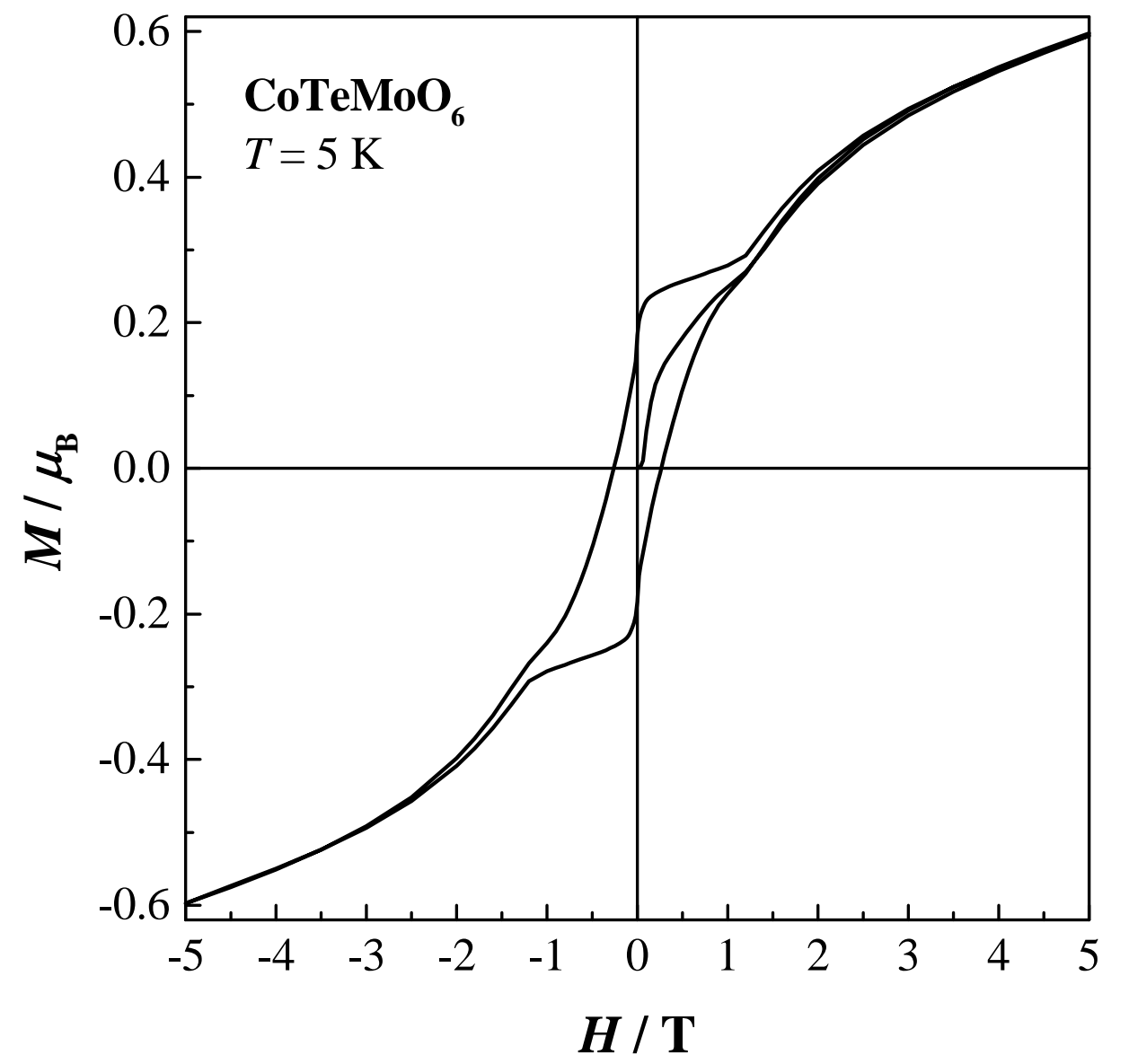

Figure 8 Doi et. al 


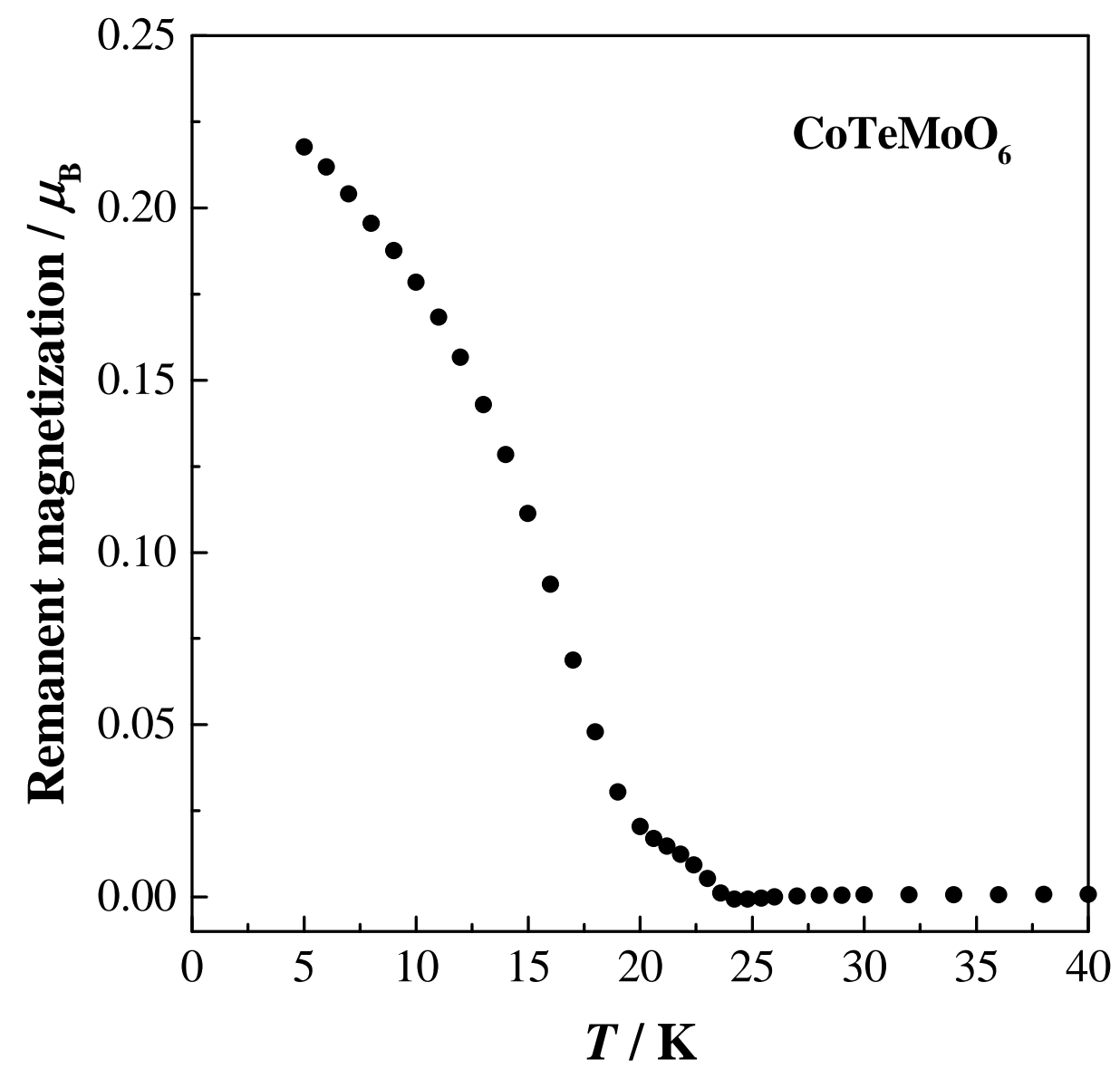

Figure 9 Doi et. al 


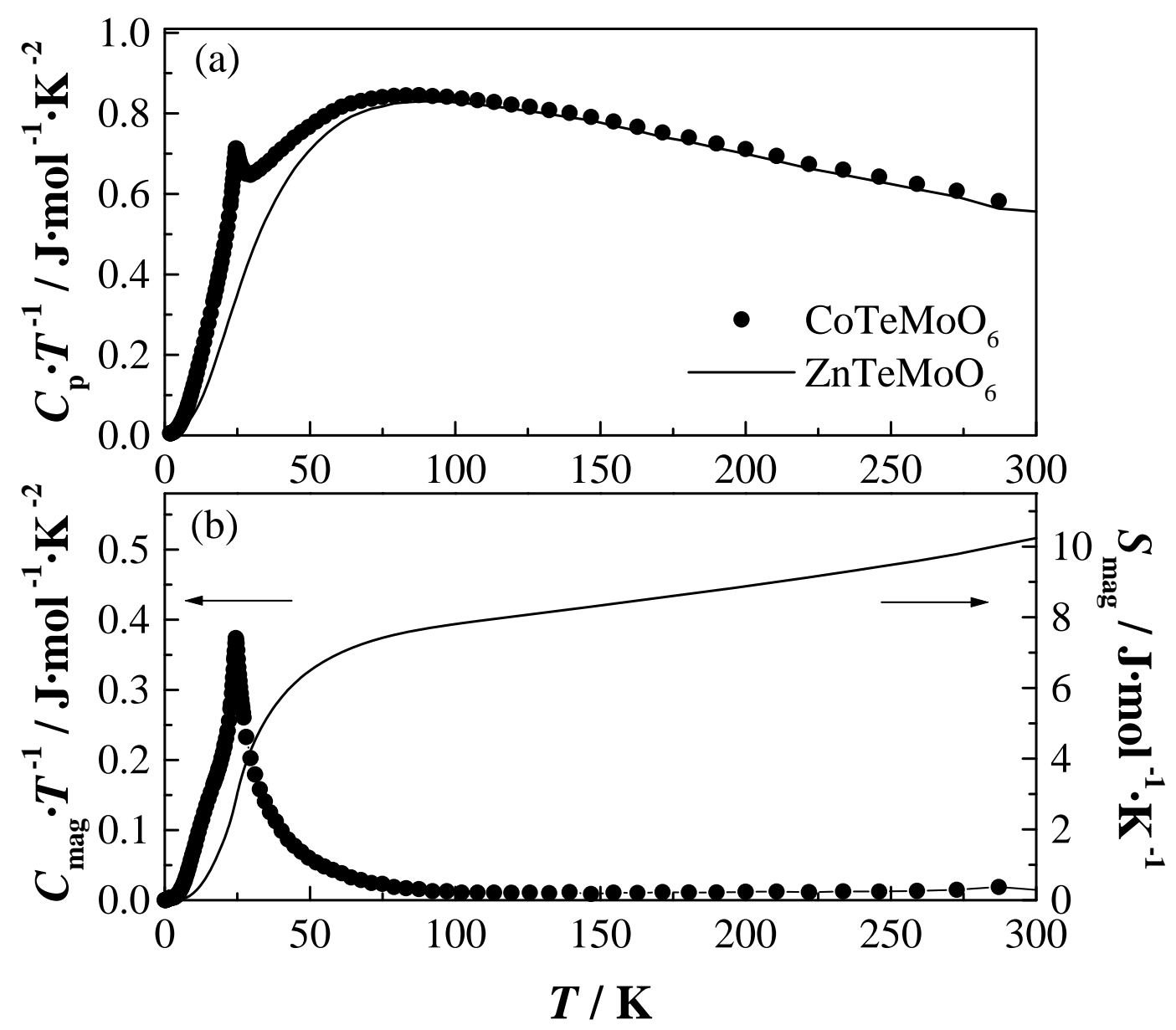

Figure 10 Doi et. al 

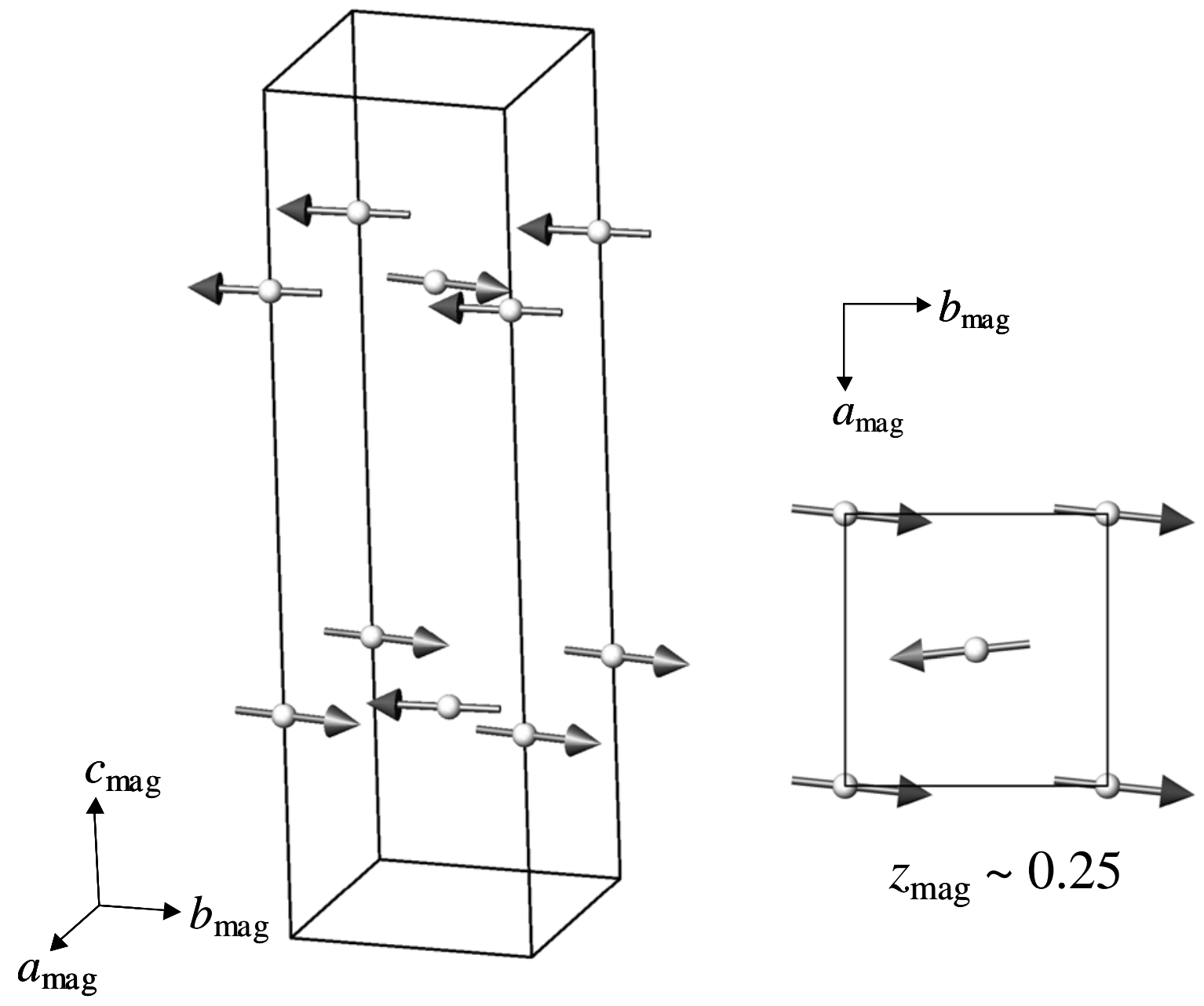

Figure 11 Doi et. al 